\title{
Fast Prototype Based Noise Reduction
}

\author{
Kajsa Tibell ${ }^{1}$, Hagen Spies ${ }^{1}$, and Magnus Borga ${ }^{2}$ \\ 1 Sapheneia Commercial Products AB, \\ Teknikringen 8, 58330 Linkoping, Sweden \\ 2 Department of Biomedical Engineering, \\ Linkoping University, Linkoping, Sweden \\ \{kajsa.tibell, hagen.spies\}@scpab.eu, \\ magnus@imt.liu.se
}

\begin{abstract}
This paper introduces a novel method for noise reduction in medical images based on concepts of the Non-Local Means algorithm. The main objective has been to develop a method that optimizes the processing speed to achieve practical applicability without compromising the quality of the resulting images. A database consisting of prototypes, composed of pixel neighborhoods originating from several images of similar motif, has been created. By using a dedicated data structure, here Locality Sensitive Hashing (LSH), fast access to appropriate prototypes is granted. Experimental results show that the proposed method can be used to provide noise reduction with high quality results in a fraction of the time required by the Non-local Means algorithm.
\end{abstract}

Keywords: Image Noise Reduction, Prototype, Non-Local.

\section{Introduction}

Noise reduction without removing fine structures is an important and challenging issue within medical imaging. The ability to distinguish certain details is crucial for confident diagnosis and noise can obscure these details. To dissolve this problem some noise reduction method is usually applied. However, many of the existing algorithms assume that noise is dominant for high frequencies and that the image is smooth or piecewise smooth when, unfortunately, many fine structures in images correspond to high frequencies and regular white noise has smooth components. This can cause unwanted loss of detail in the image.

The Non-Local Means algorithm, first proposed in 2005, addresses this problem and has been proven to produce state-of-the-art results compared to other common techniques. It has been applied to medical images (MRI, 3D-MRI images) [12] 1] with excellent results. Unlike existing techniques, which rely on local statistics to suppress noise, the Non-Local Means algorithm processes the image by replacing every pixel by the weighted average of all pixels in that image having similar neighborhoods. However, its complexity implies a huge computational burden which makes the processing take unreasonably long time. Several improvements have been proposed (see for example [1] 3] [13]) to increase the speed, but they are still too slow for practical applications. Other related methods include Discrete Universal Denoising (DUDE) proposed by Weissman et al 
[11] and Unsupervised Information-Theoretic, Adaptive filtering (UINTA) by Awate and Whitaker [10].

This work presents a method for reducing noise based on concepts of the Non-Local Means algorithm with dramatically reduced processing times. The central idea is to take advantage of the fact that medical images are limited in the matter of motif and that there already exists a huge amount of images for different kinds of examinations, and perform as much of the computations as possible prior to the actual processing.

These ideas are implemented by creating a database of pixel neighborhood averages, called prototypes, originating from several images of a certain type of examination. This database is then used to process any new image of that type of examination. Different databases can be created to provide the possibility to process different images. During processing, the prototypes of interest can be rapidly accessed, in the appropriate database, using a fast nearest neighbor search algorithm, here the Locality Sensitive Hashing (LSH) is used. Thus, the time spent on processing an image is dramatically reduced. Other benefits of this approach are that a lot more neighborhoods can contribute to the estimation of a pixel and the algorithm is more likely to find at least one neighborhood in the more unusual cases.

The outline of this paper is as follows. The theory of the Non-Local Means algorithm is described in Section 2 and the proposed method is described in Section 3. The experimental results are presented and discussed in Section 4 and finally conclusions are drawn in Section 5.

\section{Summary of the Non-local Means Algorithm}

This chapter recalls the basic concept upon which the proposed method is based.

The Non-Local means algorithm was first proposed by Buades et al. 2] in 2005 and is based on the idea that the redundancy of information in the image under study can be used to remove noise. For each pixel in the image the algorithm selects a square window of surrounding pixels with size $(2 d+1)^{2}$ where $\mathrm{d}$ is the radius. This window is called the neighborhood of that pixel. The restored value of a pixel, $i$, is then estimated by taking the average of all pixels in the image weighted depending on the similarity between their neighborhood and the neighborhood of $i$.

Each neighborhood is described by a vector $v\left(N_{i}\right)$ containing the gray level values of the pixels of which it consists. The similarity between two pixels $i$ and $j$ will then depend on the similarity of the intensity gray level vectors $v\left(N_{i}\right)$ and $v\left(N_{j}\right)$. This similarity is computed as a Gaussian weighted Euclidean distance $\left\|v\left(N_{i}\right)-v\left(N_{j}\right)\right\|_{2, a}^{2}$ which is a standard $L_{2}$-norm convolved with a Gaussian kernel of standard deviation $a$.

As described earlier the pixels need to be weighted so that pixels with a similar neighborhood to $v\left(N_{i}\right)$ are assigned larger weights on the average. Given the distance between the neighborhood vectors $v\left(N_{i}\right)$ and $v\left(N_{j}\right)$, the weight, $w(i, j)$ is computed as follows: 


$$
w(i, j)=\frac{1}{Z(i)} e^{-\frac{\left\|v\left(N_{i}\right)-v\left(N_{j}\right)\right\|_{2, a}^{2}}{h^{2}}}
$$

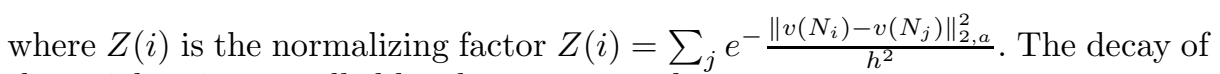
the weights, is controlled by the parameter $h$.

Given a noisy image $v=v(i)$ defined on the discrete grid $I$, where $i \in I$, the Non-Local Means filtered image is given by:

$$
N L(v)(i)=\sum_{j \in I} w(i, j) v(j),
$$

where $v(j)$ is the intensity of the pixel $j$ and $w(i, j)$ is the weights assigned to $v(j)$ in the restoration of the pixel $i$.

Several attempts have been made to reduce the computational burden related to the Non-Local Means. Already when introducing the algorithm in the original paper 2, the authors emphasized the problem and proposed some improvements. For example, they suggested to limit the comparison of neighborhoods to a so called "search window" centered at the pixel under study. Another suggestion they had was "Blockwise implementation" where the image is divided into overlapping blocks. A Non-Local Means-like restoration of these blocks is then performed and finally the pixel values are restored based on the restored values of the blocks that they belong to. Examples of other improvements are "Pixel selection" proposed by Mahmoudi and Sapiro in [3] and "Parallel computation" and a combination of several optimizations proposed by Coup et al in [1].

\section{Noise Reduction Using Non-local Means Based Prototype Databases}

Inspired by the previously described Non-Local Means algorithm and using some favorable properties of medical images a method for fast noise reduction of CT images has been developed. The following key aspects were used:

1. Create a database of pixel neighborhoods originating from several similar images.

2. Perform as much of the computations as possible during preprocessing, i.e. during the creation of the database.

3. Create a data structure that provides fast access to prototypes in the database.

\subsection{Neighborhood Database}

As described earlier, CT images are limited in terms of motif due to the technique of the acquisition and the restricted number of examination types. Furthermore, several images of similar motif already exist in medical archiving systems. This implies that it is possible to create a system that uses neighborhoods of pixels from several images. 
A database of neighborhoods that can be searched when processing an image is constructed as follows.

As in the Non-Local Means algorithm, the neighborhood $n(i)$ of a pixel $i$ is defined as a window of arbitrary radius surrounding the pixel $i$.

Let $N_{I}$ be a number of images of similar motif with size $I^{2}$. For every image $I_{1 \ldots N_{I}}$ extract the neighborhoods $n(i)_{1, \ldots, I^{2}}$ of all pixels $i_{1, \ldots, I^{2}}$ in the image. Store each extracted neighborhood as a vector $v(n)$ in a database. The database $D(v)$ will then consist of $S_{D}=N_{I} * I^{2}$ neighborhood vectors $v(n)_{1, \ldots, S_{D}}$ :

$$
D(v)=v(n)_{1, \ldots, S_{D}}
$$

\subsection{Prototypes}

Similar to the blockwise implementation suggested in [2] the idea is to reduce the number of distance and average computations performed during processing by combining neighborhoods. The combined neighborhoods are called prototypes. Then the pixel values can be restored based on the values of these prototypes.

If $q(n)$ is a random neighborhood vector stored in the database $D(v)$ a prototype is created by computing the average of the neighborhood vectors $v(n)$ at distance at most $w$ from $q(n)$.

By randomly selecting $N_{p}$ number of neighborhood vectors from the database and compute the weighted average for each of them the entire database can be altered so that all neighborhood vectors are replaced by prototypes. The prototypes are given by:

$$
P(v)_{1, \ldots, N_{p}}=\frac{1}{C_{i}} \sum_{i \in D} v(n)_{i} \text { if }\left\|q(n)-v(n)_{i}\right\|_{2}^{2}<w
$$

where $C_{i}=\sum_{i \in D} v(n)_{i}$. Clearly, the number of prototypes in the database will be much smaller than the number of neighborhood vectors. Thus, the number of similarity comparisons during processing is decreased. However, for fast processing the relevant prototypes need to be accessed without having to search through the whole database.

\subsection{Similarity}

The neighborhood vectors can be considered to be feature vectors of each pixel of an image. Thus, they can be represented as points in a feature space with the same dimensionality as the size of the neighborhood. The points that are closest to each other in that feature space are also the most similar neighborhoods. Finding a neighborhood similar to a query neighborhood then becomes a Near Neighbor problem (see [9] [5] for definition).

The prototypes are, as described earlier, restored neighborhoods and thereby also points living in the same feature space as the neighborhood vectors. They are simply points representing a collection of the neighborhood vector points that lie closest to each other in the feature space.

As mentioned before, the Near Neighbor problem can be solved by using a dedicated data structure. In that way linear search can be avoided and replaced by fast access to the prototypes of interest. 


\subsection{Data Structure}

The data structure chosen is the Locality Sensitive Hashing (LSH) scheme proposed by Datar et al [6] in 2003 which uses p-stable distributions [8] 7] and works directly on points in Euclidean space. Their version is a further development of the original scheme introduced by P. Indyk and R. Motwani [5] in 1998 whose key idea was to hash the points in a data set using hash functions such that the probability of collision is much higher for points which are close to each other than for points that are far apart. Points that collide are collected in "buckets" and stored in hash tables. The type of functions used to hash the points belong to what is called a locality-sensitive hash (LSH) family.

For a domain $S$ of the point set with distance $D$, an locality-sensitive hash (LSH) family is defined as:

Definition 1. A family $H=h: S \rightarrow U$ is called locality-sensitive (or $\left(r_{1}, r_{2}, p_{1}, p_{2}\right)$-sensitive) for $D$ if for any $v, q \in S$

- if $v \in B\left(q, r_{1}\right)$ then $\operatorname{Pr}_{H}[h(q)=h(v)] \geq p_{1}$

- if $v \notin B\left(q, r_{2}\right)$ then $\operatorname{Pr}_{H}[h(q)=h(v)] \leq p_{2}$

where $r_{1}=R$ and $r_{2}=c * R, B(q, r)$ is a ball of radius $r$ centered in $q$ and $\operatorname{Pr}_{H}[h(q)=h(v)]$ is the probability that a point $q$ and a point $v$ will collide if using a hash function $h \in H$. The LSH family has to satisfy the inequalities $p_{1}>p_{2}$ and $r_{1}<r_{2}$ in order to be useful. By using functions from the LSH family the set of points can be preprocessed so that adjacent points are stored in the same bucket. When searching for the neighbors of a query point $q$ the same functions are used to compute which "bucket" shall be considered. Instead of the whole set of points, only the points inside that "bucket" need to be searched.

The LSH algorithm was chosen since it has proven to have better query time than spatial data structures, the dependency on dimension and data size is sublinear and it is somewhat easy to implement.

\subsection{Fast Creation of the Prototypes}

As described in 3.2 a prototype is created by finding all neighborhood vectors similar to a randomly chosen neighborhood in the database and computing their average. To achieve fast creation of the prototypes the LSH data structure is applied. Given a number $N_{I}$ of similar images the procedure is as follows: First all neighborhoods $n(i)_{1, \ldots, I^{2}}$ of the first image are stored using the LSH data structure described above. Next, a random vector is chosen and used as a query $q$ to find all similar neighborhood vectors. The average of all neighborhood vectors at distance at most $w$ from the query is computed producing the prototype $P(v)_{i}$. The procedure is repeated until an chosen number $N_{p}$ of prototypes is created. Finally all neighborhood vectors are deleted from the hash tables and the prototypes $P(v)_{1, \ldots, N_{p}}$ are inserted instead. For all subsequent images every neighborhood vector is used as a query searching for similar prototypes. If a prototype is found the neighborhood vector is added to that by computing the average of the prototype and the vector itself. Since a prototype $P(v)_{i}$ most 
often is created of several neighborhood vectors and the query vector $q$ is single, the query vector should not have equal impact on the average. Thus, the average has to be weighted by the number of neighborhood vectors included.

$$
P(v)_{i N e w}=\frac{P(v)_{i} * N_{v}+q}{N_{v}+1}
$$

where $N_{v}$ is the number of neighborhood vectors that the prototype $P(v)_{i}$ is composed of. If for some query vector no prototype is found that query vector will constitute a new prototype itself. Thereby, unusual neighborhoods will still be represented.

\subsection{The Resulting Pipeline}

The resulting pipeline of the proposed method consist of two phases. The preprocessing phase where a database is created and stored using the LSH scheme and the processing phase where the algorithm reduces the noise in an image using the information stored in the database.

Creating the Database. First the framework of the data structure is constructed. Using this framework the neighborhood vectors $v(n)_{i}$ of $N_{I}$ similar images are transformed into prototypes. The prototypes $P(v)_{i N e w}$, which constitutes the database, are stored in "buckets" depending on their location in the high dimensional space in which they live. The "buckets" are then stored in hash tables $T_{1}, \ldots, T_{L}$ using a universal hash function, see fig 1 .

Processing an Image. For every pixel in the image to be processed a new value is estimated using the prototypes stored in the database. By utilizing the data structure the prototypes to be considered can be found simply by calculating the "buckets" $g_{1}, \ldots, g_{L}$ corresponding to the neighborhood vector of the pixel under process and the indexes of those "buckets" in the hash tables $T_{1}, \ldots, T_{L}$. If more than one prototype is found the distance to each prototype is computed. The intensity value $p(i)$ of the pixel $i$ is then estimated by interpolating the prototypes $P(v)_{k}$ that lies within radius $s$ from the neighborhood $v(n)_{i}$ of $i$ using inverse distance weighting (IDW).

Applying the general form of the IDW using a weight function defined by Shepard in [4] gives the expression for the interpolated value $p(i)$ of the point $i$ :

$$
p(i)=\frac{\sum_{k \in N_{p}} w(i)_{k} P(v)_{k}}{\sum_{k \in N_{p}} w(i)_{k}}
$$

where $w(i)_{k}=\frac{1}{\left(\left\|v(n)_{i}-P(v)_{k}\right\|_{2}^{2}\right)^{t}}, N_{p}$ is the number of prototypes in the database and $t$ is a positive real number, called the power parameter. Greater values of $t$ emphasizes the influence of the values closest to the interpolated point and the most common value of $t$ is 2 . If no prototype is found the original value of the pixel will remain unmodified. 


\section{Creating the database}
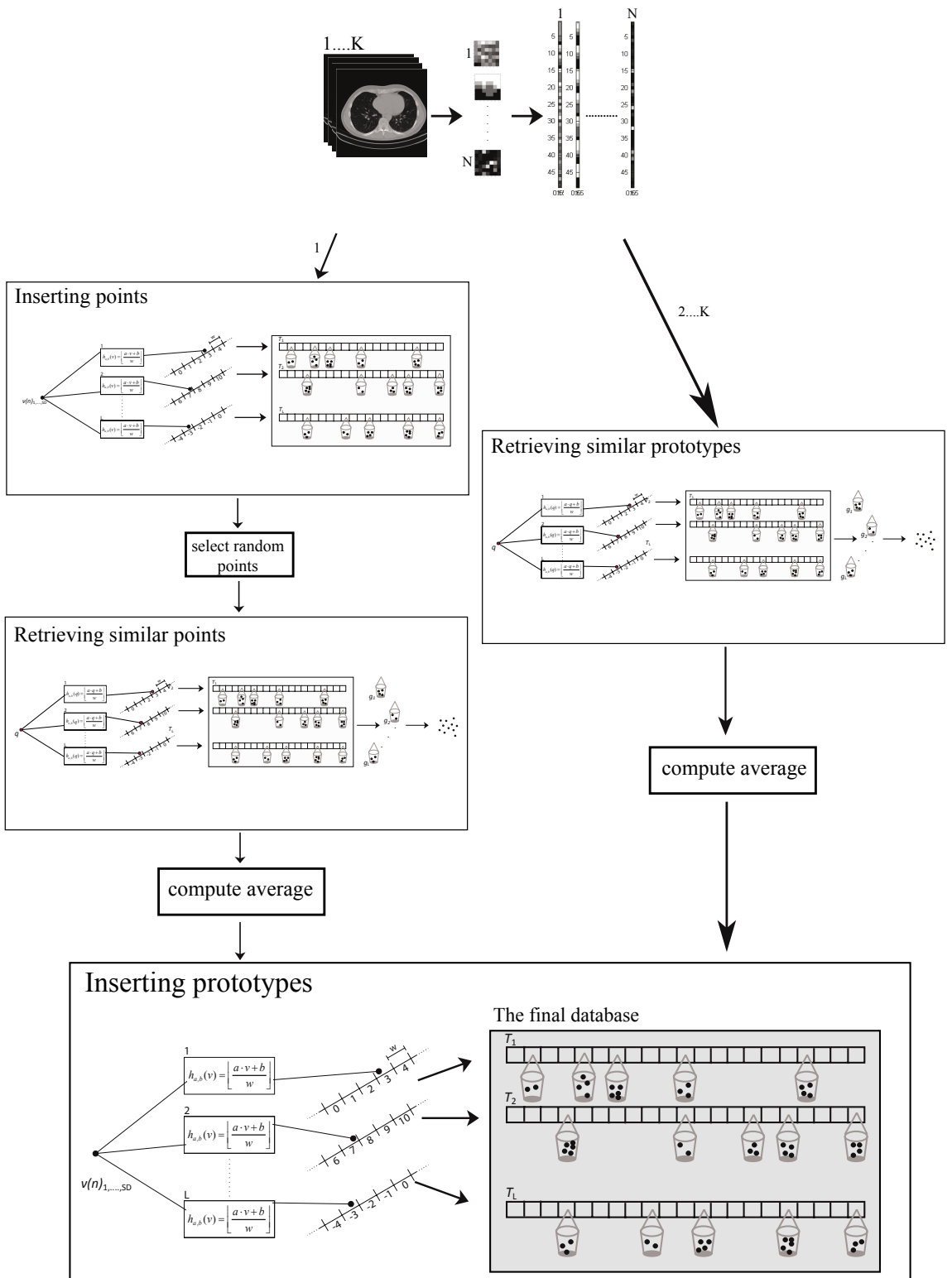

Fig. 1. A schematic overview of the creation of a database 

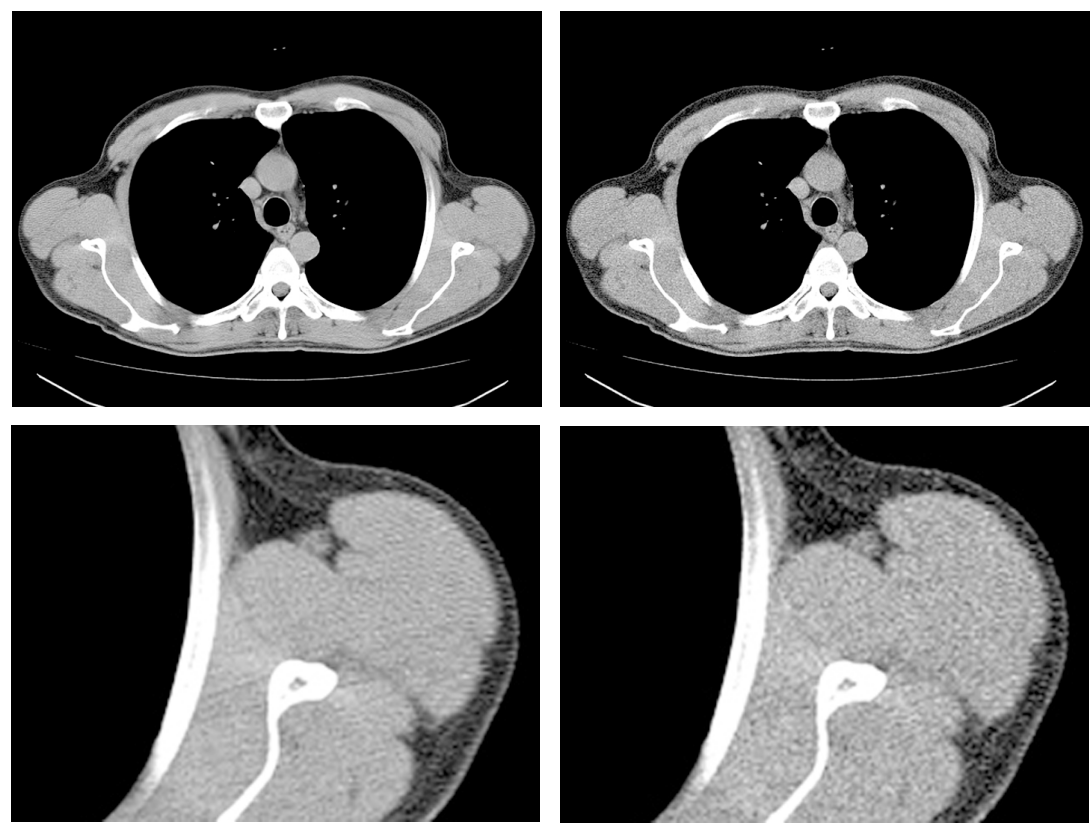

Original Image

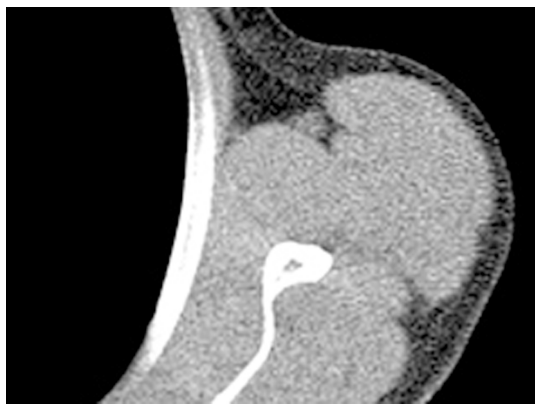

Noise Image
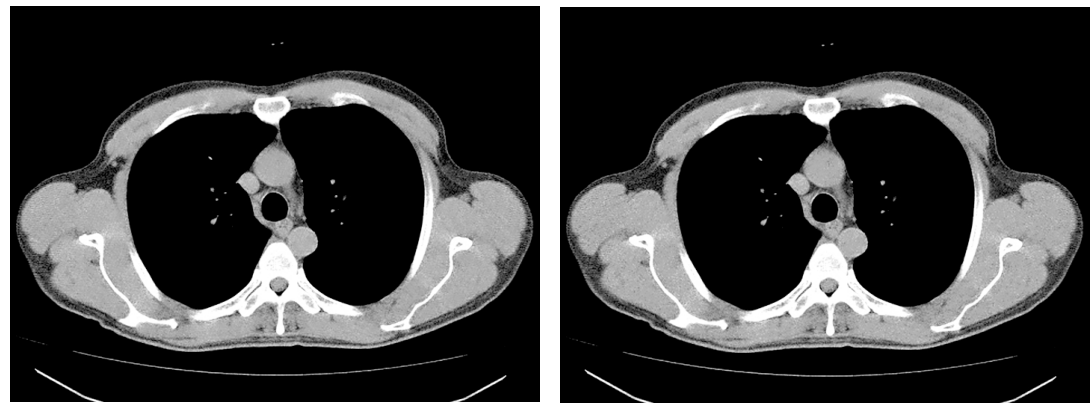

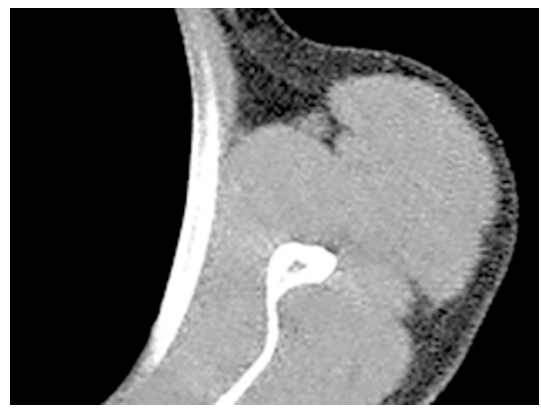

Proposed Algorithm

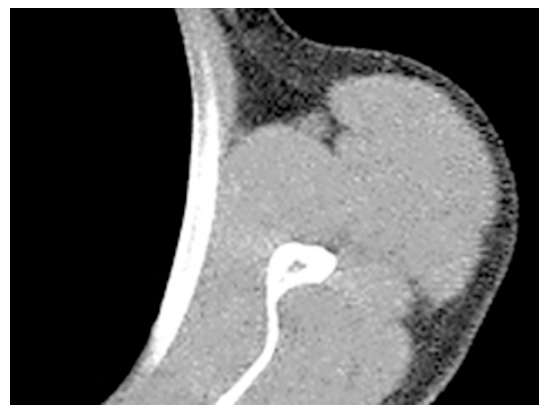

Non-Local Means

Fig. 2. CT image from lung with enlarged section below 


\section{Experimental Results}

To test the performance of the proposed algorithm several databases have been created using different numbers of images. As expected, increasing the number of images used also increased the quality of the resulting images. The database used for processing the images in fig 2 consisted of 48772 prototypes obtained from the neighborhoods of 17 similar images. Two sets of images were tested one of which is presented here. White Gaussian noise was applied to all images in one of the test sets (presented here) and the size of the neighborhoods was set to $7 * 7$ pixels.

The results was compared to The Non-Local Means algorithm and to evaluate the performance of the algorithms, quantitatively, the peak-to-peak signal to noise ratio (PSNR) was computed.

Table 1. PSNR and processing times for the test images

\begin{tabular}{|c|c|c|}
\hline Method & PSNR & Time $(s)$ \\
\hline Non-Local Means & 126.9640 & 34576 \\
Proposed method & 129.9270 & 72 \\
\hline
\end{tabular}

The results in fig 2 shows that the proposed method produces an improved visual result compared to the Non-Local Means. The details in the resulting image are better preserved while a high level of noise reduction is still maintained. Table 1 shows the PSNR and processing times obtained.

\section{Conclusions and Future Work}

This paper introduced a noise reduction approach based on concepts of the Non-Local Means algorithm. By creating a well-adjusted database of prototypes that can be rapidly accessed using a dedicated data structure it was shown that a noticeably improved result can be achieved in a small fraction of the time required by the existing Non-Local Means algorithm. Some further improvement in the implementation will enable using the method for practical purposes and the presented method is currently being integrated in the Sapheneia Clarity product line for low dose CT applications.

Future work will include investigation of alternative features, of the neighborhoods, replacing the currently used intensity values. Furthermore, the dynamic capacity of the chosen data structure will be utilized for examining the possibility to continuously integrate the neighborhoods, of the images being processed, into the database for making it adaptive.

\section{References}

1. Coupe, P., Yger, P., Prima, S., Hellier, P., Kervrann, C., Barillot, C.: An Optimized Blockwise Nonlocal Means Denoising Filter for 3-D Magnetic Resonance Images. IEEE Transactions on Medical Imaging 27(4), 425-441 (2008) 
2. Buades, A., Coll, B., Morel, J.M.: A review of image denoising algorithms, with a new one. Multiscale Modeling \& Simulation 4(2), 490-530 (2005)

3. Mahmoudi, M., Sapiro, G.: Fast image and video denoising via nonlocal means of similar neighborhoods. IEEE Signal Processing Letters 12(12), 839-842 (2005)

4. Shepard, D.: A two-dimensional interpolation function for irregularly-spaced data. In: Proceedings of the 1968 ACM National Conference, pp. 517-524 (1968)

5. Indyk, P., Motwani, R.: Approximate nearest neighbor: towards removing the curse of dimensionality. In: Proceedings of the 30th Symposium on Theory of Computing, pp. 604-613 (1998)

6. Datar, M., Immorlica, N., Indyk, P., Mirrokni, V.: Locality-sensitive hashing scheme based on p-stable distributions. In: DIMACS Workshop on Streaming Data Analysis and Mining (2003)

7. Nolan, J.P.: Stable Distributions - Models for Heavy Tailed Data. Birkhäuser, Boston (2007)

8. Zolotarev, V.M.: One-Dimensional Stable Distributions. Translations of Mathematical Monographs 65 (1986)

9. Andoni, A., Indyk, P.: Near-Optimal hashing algorithm for approximate nearest neighbor in high dimensions. Communications of the ACM 51(1) (2008)

10. Awate, S.A., Whitaker, R.T.: Image denoising with unsupervised, informationtheoretic, adaptive filtering. In: Proceedings of the IEEE International Conference on Computer Vision and Pattern Recognition (2005)

11. Weissman, T., Ordentlich, E., Seroussi, G., Verdu, S., Weinberger, M.: Universal discrete denoising: Known channel. IEEE Transactions on Information Theory 51, $5-28(2005)$

12. Manjón, J.V., Carbonell-Caballero, J., Lull, J.J., García-Martía, G., MartíBonmatíb, L., Robles, M.: MRI denoising using Non-Local Means. Medical Image Analysis 12, 514-523 (2008)

13. Wong, A., Fieguth, P., Clausi, D.: A Perceptually-adaptive Approach to Image Denoising using Anisotropic Non-Local Means. In: The Proceedings of IEEE International Conference on Image Processing (ICIP) (2008) 